\title{
A signal-detection approach to subception: Concomitant verbal and finger-latency responses in metacontrast
}

\author{
DANIEL J. WEINTRAUB \\ The University of Michigan, Ann Arbor, Michigan 48104 \\ and \\ LINDA S. FIDELL \\ California State University, Northridge, California 91330
}

\begin{abstract}
Metacontrast conditions were created by the onset of flanking lights designed to mask the prior blink of an otherwise steady center light. For pseudometacontrast trials, the center light did not blink in advance of the flanking lights. Responses were an immediate fingerkey release to the first detectable change in the visual display followed by a statement of whether the center light had been doused. A signal-detection analysis was used to avoid both threshold and response-criterion problems. Verbal report was, on the average, more sensitive than finger latency in detecting the masked blinks. However, there were large and consistent individual differences; reaction time was the more sensitive for a few subjects. Data analysis revealed that each response system showed detection with the other system "partialed out." A model was offered in which verbal- and finger-response systems act in parallel, with uncorrelated variability between systems accounting for the subception effect.
\end{abstract}

The extensive literature concerned with subliminal perception (subception, "perceiving"' below the level of awareness) demonstrates both the importance of such a construct and the difficulties involved in its validation. Ours is a rescue mission, to save a traditional-and rigorous-experimental paradigm, the comparison of nonverbal with verbal responses, from known perils, pressing toward the answers these studies were designed to reveal. We emphasize that metacontrast (backward temporal masking of one luminous region by a surrounding luminous region), dual-response performance, and the theory of signal detectability are treated only as means to an end, namely, the understanding of subliminal perception.

Lazarus and McCleary (1951) defined subception as "a process by which some kind of discrimination

The investigation was supported in part by a United States Public Health Service Research Scientist Development Award (K2-MH-35,253) to the first author, and USPHS Fellowship 1-F1MH-29,060-01 to the second author. Manuscript preparation was supported by Grant MH23767. This support was provided by the National Institute of Mental Health. Research funds were provided by National Science Foundation Grant GB 3110, and a Rackham grant from the University of Michigan. The authors sincerely thank J. E. K. Smith for the insights contributed toward unraveling the complexities of the data, and S. R. Doehrman and J. D. Chananie for their long-term efforts in the frustrating and fruitless search for a signal-detection subception design incorporating a functioning GSR detection system. Requests for reprints should be sent to Daniel J. Weintraub, Human Performance Center, Perry Building, 330 Packard Road, Ann Arbor, Michigan 48104. is made when the subject is unable to make a correct conscious discrimination"' (p. 113). Comparisons between response systems under conscious and unconscious control have been crucial in attempts to provide an empirical test of discrimination without awareness. Verbalizability is the usual operational criterion for awareness; a perceiver is aware of what s(he) can report. A perceiver is presumed, therefore, not to be aware of autonomic responses, such as changes in skin potential (GSR). Simple reaction time, a sensitive and reliable response, might distinguish between stimuli and also meet a consensus criterion of unawareness. We propose that subjects in the metacontrast experiment described below, although well aware that they are responding, are unaware of the small latency differences, less than $20 \mathrm{msec}$ on the average, between their responses to stimulus-present and stimulus-absent trials, i.e., between metacontrast and pseudometacontrast. These small time differences would not seem to be amenable to conscious monitoring or modulation. Metacontrast is a useful phenomenon for the study of subception because there is good reason to believe that, on many trials, subjects are truly unaware of the stimulus event they are asked to detect.

The classical metacontrast experiment (Raab, 1963) involves spatially contiguous lights. The brightness of a briefly presented center light is markedly reduced if flanking lights (the mask) appear within 100 msec. Schiller and Smith (1966) reported that 
simple reaction time, the time to make a finger response as rapidly as possible after the onset of the center light, seemed unaffected by the introduction of the mask even though the accuracy of verbal reports about the presence of the stimulus was degraded to near-chance levels. Although the brightness (apparent intensity) of the center light decreases toward zero, the presence of the center light can often be inferred from apparent movement or other perceptual cues (Fehrer \& Raab, 1962).

Fehrer and Biederman (1962) redesigned the metacontrast experiment to make the detection task more difficult. The center light remained on except for a 5 -msec blink (absence) that was the signal to be detected. The flanking lights then masked the blink. With both highly practiced and naive subjects, they found that a $30-\mathrm{msec}$ stimulus-onset delay between the blink of the center light and the occurrence of the flanking lights affected mean simple reaction time to the blink minimally while the accuracy of verbal report was about $67 \%$. Our experiment utilized the metacontrast design of Fehrer and Biederman with a modification, concomitant responses, in order to investigate the mechanisms of subception. A simple finger-withdrawal response followed by a verbal report concerning whether the center light had blinked was required on every trial. The flash of flanking lights is a salient event that cannot pass undetected, but a finger response can be initiated even sooner by the preceding offset of the center light on trials when it is detected. Therefore, a rapid finger response can be accepted as the nonverbal detection of a blink, and compared with the verbal report of the same event.

Eriksen $(1956,1960)$ reformulated the subception effect as a correlation problem: Each of the two responses mirrors (i.e., is correlated with) the stimulus - but not perfectly-and the responses are correlated -but not perfectly-with each other. By obtaining both responses to every occurrence of the stimulus, the experimenter can determine response-response correlations in addition to stimulus-response correlations. The correlations and partial correlations derived from a concomitant-responses experimental design permit evaluation of the various classes of subception theories proposed by Eriksen.

The signal-detectability approach has two salient advantages. It bypasses threshold problems, and it resolves the response-criterion dilemma by considering hits (correct detections) in relation to false alarms (incorrect detections). (Consult, also, the discussion by Bernstein, Amundson, \& Schurman, 1973). Verbal reports give estimates of the probability of reporting the masked blink of the center light when it occurred (metacontrast), i.e., the hit rate (HR), and the probability of reporting the masked blink when it did not occur (pseudometacontrast), i.e., the false-alarm rate
(FAR). The obtained FAR-HR pair provides a detectability index, a point on a receiver operating characteristic (ROC) curve. Reaction times provide two distributions: reaction times to masked blinks and reaction times to flanking lights alone (see the sketch in Figure 1). A criterion can arbitrarily be chosen, and for all latencies shorter than the criterion the decision is taken to be "masked blink." Each decision criterion provides one FAR-HR pair; the set of all pairs defines the ROC curve. The performance of response systems can be compared by comparing ROC curves. Eriksen $(1956,1960)$ noted that restricting verbal report to a limited number of response categories may severely handicap the expression of the richness and detail of the perceived stimulus. In the metacontrast-pseudometacontrast design consisting of only two states of the world, no matter how complex and multidimensional the stimulus, one can argue from theoretical assumptions that no detection capacity is sacrificed by requiring a simple yes or no detection response. (More precisely, and more technically, no capacity is sacrificed if decisions are based on likelihood-ratio criteria. See Green \& Swets, 1966.) The procedure, however, estimates only one point on the ROC curve, which is a distinct drawback. Although verbal report in a signal-detectability analysis is not disadvantaged by response restrictions, it may well be at a disadvantage because of the necessity to reach a decision. For example, inability to maintain an invariant decision criterion will degrade performance. Reaction time, on the other hand, makes no decisions; the experimenter, in effect, generates decisions from the data. Her arbitrary choice of a latency criterion assigns every latency either to the detect or the nondetect response category.

In summary, subception (subliminal perception) was investigated using the metacontrast paradigm of Fehrer and Biederman (1962), which incorporates many features advantageous to study of subception, and a signal-detection analysis of the data (Green \& Swets, 1966; Swets, Tanner, \& Birdsall, 1961), which has achieved considerable success in separating response-criterion problems from indices of sensitivity. We build on Bernstein, Amundson, and Schurman's (1973) astute signal-detection approach to the Fehrer and Biederman (1962) design, and on the insights of Eriksen $(1956,1957)$ with respect to the interpretation of correlations and partial correlations among stimuli and responses in subception experiments. To these are added the keystone, concomitant responding.

\section{EXPERIMENT 1}

\section{Method}

Each of four adult subjects participated in one experimental session per day for 8 days, the first 4 considered as practice. 
Subjects binocularly viewed a display $89 \mathrm{~cm}$ away at the end of a dark tunnel. The display consisted of three neon bulbs (GE2P) behind three contiguous vertical rectangular bars (each $12.7 \times$ $31.8 \mathrm{~mm}$ ) of translucent plastic, each bar yielding a luminance of $60 \mathrm{~cd} / \mathrm{m}^{2}$, as measured by a Macbeth illuminometer. The bulbs have negligible rise and fall times relative to the response latencies under consideration and an orange hue. The total display subtended $3^{\circ}$ of visual angle horizontally and $2.4^{\circ}$ vertically $(38.1 \times 31.8 \mathrm{~mm})$.

A muffled click whose onset was difficult to pinpoint marked the beginning of a trial; one of four possible stimulus configurations then appeared (see the inset in Figure 1). During a centerplus-flanks $(C+F)$ metacontrast trial, 2,000 msec after the click, the center light, which remained on continuously between trials, went off for $5 \mathrm{msec}$. Then, $35 \mathrm{msec}$ after the offset of the center light ( $30 \mathrm{msec}$ after its return), both flanking lights appeared for 100 msec. A flanks (F) pseudometacontrast trial differed only in that the center light never blinked; the flanking lights still appeared $2,035 \mathrm{msec}$ after the click. During a center (C) trial, the center light blinked off for $5 \mathrm{msec}$ and the two flanking lights never came on. In a blank (B) trial, there was no change in the stimulus configuration; the center light simply stayed on, the flanking lights off.

Each day the subject was shown 10 preliminary trials with the type of trial identified for the subject preceding each presentation. Twenty practice-with-feedback trials followed during which the subject was told the correct stimulus configuration after responding. On each day of the main experiment, three blocks of 109 trials per block were administered, $38 \mathrm{C}+\mathrm{F}, 38 \mathrm{~F}, 22 \mathrm{C}$, and $11 \mathrm{~B}$ trials, the trials randomly ordered within a block.

The instructions were to respond by removing the index finger of the preferred hand from a telegraph key as quickly as possible after the first detectable change in the visual stimulus configuration. (The flanking lights almost always elicit a finger response, although a preceding blink may not.) Reaction times were measured from $2 \mathrm{sec}$ after the auditory cue regardless of type of trial, and were recorded to the nearest $.01 \mathrm{sec}$. On a concomitantresponse trial, after completing each finger withdrawal the subject responded verbally with a confidence rating chosen from one of four categories: Yes + ("Yes. I am very sure the center light went off."), Yes ("Yes. I think the center light went off."), No ("No. I don't think the center light went off."), or No+ ("No. I am very sure the center light did not go off."). The subjects responded during the other two daily blocks by providing either reaction times alone or verbal reports alone. The order of blocks was counterbalanced across days for each subject.

\section{Results}

Figure 1 displays the detectability data. The threepoint estimates comprising an ROC curve for verbal report are shown. By means of successive criterion cuts through the overlapping reaction-time distributions to $C+F$ and $F$ stimuli, treating the shorter latencies as reported detections, an empirical ROC curve for finger withdrawal was generated. (The depicted functions have been smoothed by connecting the nearest points for which both false-alarm rate and hit rate differ.) Detection differences can be interpreted directly. If all point estimates for verbal report lie above the ROC curve for reaction time (greater hit rate for the same false-alarm rate), then verbal report is the more sensitive system; if the points lie below, then verbal report is less sensitive. Under concomitant responding (RT \& VR, Figure 1), verbal report is the more sensitive for two subjects, reaction

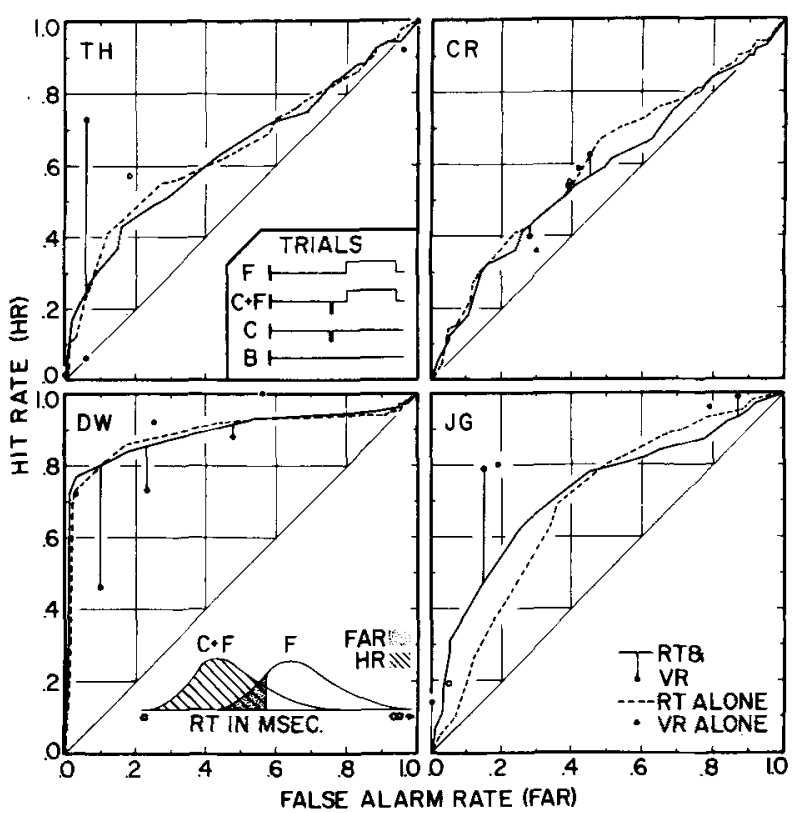

Figure 1. Detection data for Experiment 1. The inset, upper left, shows stimulus conditions. The sketch, lower left, depicts schematically the overlapping reaction-time distributions from which ROC curves are derived.

time is more sensitive for one, and there is essentially a tie for one subject. The comparison between concomitant and nonconcomitant responding indicates little or no difference in finger-withdrawal detectabilities. However, for verbal report, one subject improved when no finger response was required, another suffered a decrement, and two were basically unchanged.

Statistical tests confirm what can be gleaned by eye from Figure 1. Differences between mean reaction times calculated from latencies on $C+F$ trials and from those on $\mathrm{F}$ trials were subjected to a $\mathrm{z}$ test. Results appear in the RT ( $\mathrm{z}$ value) column of Table 1. The statistic, $z$, is related to the signal-detectability measure, $d^{\prime}$. If one were to assume that the two response populations were normally distributed with equal variance, then $d^{\prime}=z \sqrt{\left(N_{1}+N_{2}\right) /\left(N_{1} N_{2}\right)}$; where Ns are sample sizes of the two types of trials. One subject did not demonstrate a statistically significant detection rate for concomitant reaction-time responses. For statistical testing, verbal reports were treated as yes-no (two-category) data (which is equivalent to selecting the middle FAR-HR point). For verbal responses to the stimuli, the hit rate (defined as the probability of a "yes-either confidence category-the center light went off" response given a $\mathrm{C}+\mathrm{F}$ trial) and the false-alarm rate (the probability of a "yes ..." given an F trial) appear in the VR(FAR/ HR) column of Table 1 . The 2 by 2 contingency table generated by the responses (yes or no) to the 152 $\mathrm{C}+\mathrm{F}$ trials and $152 \mathrm{~F}$ trials was subjected to a chisquare test of independence $(1 \mathrm{df})$. For all subjects, 
Table 1

Evaluation of Response Systems for the C+F and F Trials in Experiment 1

\begin{tabular}{|c|c|c|c|c|c|c|}
\hline \multirow[b]{2}{*}{ Subject } & \multirow[b]{2}{*}{ Condition } & \multirow{2}{*}{$\begin{array}{c}\text { RT } \\
\text { (z Value) }\end{array}$} & \multirow{2}{*}{$\begin{array}{c}\text { VR } \\
\text { (FAR/HR) } \\
\end{array}$} & \multicolumn{3}{|c|}{ ROC Analysis } \\
\hline & & & & RT vs. VR & RT vs. RT & VR vs. VR \\
\hline C.R. & $\begin{array}{l}\text { RT \& VR } \\
\text { RT or VR }\end{array}$ & $\begin{array}{c}.96 \\
1.74 *\end{array}$ & $\begin{array}{l}.39 / .53^{* *} \\
.39 / .55^{* *}\end{array}$ & $\begin{array}{l}\text { n.s. } \\
\text { n.s. }\end{array}$ & n.s. & n.s. \\
\hline D.W. & $\begin{array}{l}\text { RT \& VR } \\
\text { RT or VR }\end{array}$ & $\begin{array}{l}13.87^{* *} \\
10.20^{* *}\end{array}$ & $\begin{array}{l}.23 / .74 * * \\
.25 / .92 * *\end{array}$ & $\begin{array}{l}\mathrm{RT}^{*} \\
\text { n.s. }\end{array}$ & n.s. & Alone** \\
\hline T.H. & $\begin{array}{l}\text { RT \& VR } \\
\text { RT or VR }\end{array}$ & $\begin{array}{l}3.58 * * \\
2.92 * *\end{array}$ & $\begin{array}{l}.07 / .73^{* *} \\
.18 / .57^{* *}\end{array}$ & $\begin{array}{l}\text { VR** } \\
\text { VR** }\end{array}$ & n.s. & Concomitant** \\
\hline J.G. & $\begin{array}{l}\text { RT \& VR } \\
\text { RT or VR }\end{array}$ & $\begin{array}{l}5.21 * * \\
4.22 * *\end{array}$ & $\begin{array}{l}.15 / .79 * * \\
.19 / .80^{* *}\end{array}$ & $\begin{array}{l}\text { VR** } \\
\text { VR** }\end{array}$ & n.s. & n.s. \\
\hline
\end{tabular}

Note-RT \& VR designate the concomitant-response condition. RT or VR designates the two conditions in which each response was given alone. One-tailed statistical tests were used except for the ROC analysis. Not significant is abbreviated n.s. ${ }^{*} p<.05 ;{ }^{* *} p<.01$.

verbal reports were significant predictors of the stimulus that had been presented. A statistical procedure devised by Gourevitch and Galanter (1967), based upon normality and equal-variance assumptions, permits a test to determine whether one response system was superior to the other. In order to obtain the difference measure, $G$, the point on the reaction-time ROC curve closest to the negative diagonal of the unit square was chosen for comparison with the yesno verbal-report FAR-HR point. Statistically significant differences are indicated in the table. (Our overall objective was to eschew restrictive assumptions; the use of equal-variance normal distributions served for statistical testing purposes only. Statistical analyses using differences in area under the ROC curves gave analogous results. Computing and comparing areas has its own assumptions and problems. See Bernstein et al., 1973.)

Detection of the offset of the center light in the absence of flanking lights was far better than masked detection. No subject exceeded $5 \%$ errors in either the $\mathrm{C}$ or $\mathrm{B}$ conditions, according to the verbal-report measure or finger withdrawals (for $\mathrm{C}$, not to withdraw is an error). Mean reaction times for each type of trial are listed in Table 2. Although detectability did not improve, latencies were shorter when verbal reports were not required. Reaction times to $\mathrm{C}$ are slowest.

Table 2

Mean Reaction Time (in Milliseconds) for a Concomitant Finger Response

\begin{tabular}{lccc}
\hline & C Trials & C+F Trials & F Trials \\
\hline Experiment 1 & 271 & 244 & 262 \\
Nonconcomitant & $(250)$ & $(230)$ & $(246)$ \\
Experiment 2 & None & 222 & 236 \\
\hline
\end{tabular}

Note-Times are measured from the offset of the center light or, in the case of $F$ trials, when the offset would have occurred. Data from all nondetectors are included. Numbers in parentheses are for responses in Experiment 1 when a verbal report was not given.
In summary, the substantial differences among individuals make for cautious generalizations. Subjects differ concerning the level of detection performance for each type of response, which response system is the more sensitive, and how response systems are affected by concomitant vs. nonconcomitant responding. Verbal report was not consistently superior to finger withdrawal, though it was better on the average. (For subject D.W., reaction time was superior from the earliest practice session on.) In our laboratory, all attempts to compare verbal report with various measures of shock-conditioned skin resistance on a trial-by-trial basis in signal-detection and forcedchoice experiments (cf. Dulany \& Eriksen, 1959) have failed. The problem is that, in order to obtain skinresistance detection rates minimally above chance, stimulus levels must be set in a range that produces nearly perfect verbal reporting. No additional information can be conveyed by the nonverbal response when the verbal response conveys all. A goal, then, was to obtain data in which the response presumed not to be under conscious control detected moderately well while verbal-report detection remained far from perfect. The goal was realized in this experiment.

Requiring an additional response altered detectability performance relatively little. As Figure 1 shows, reaction-time detections for concomitant vs. nonconcomitant responding are very similar for every subject. For verbal-report detection when finger-withdrawal preceded, one subject improved significantly, one performed significantly worse. Even if it is conceded that detection rates are roughly comparable, generalizing from the dual-response to the usual single-response situation is hazardous. Response latencies differ (Table 2), for example, even when detectability remains the same.

Given the variability within and between subjects, the decision was made to require more extensive practice, to obtain more concomitant data per subject, and to increase the number of subjects tested, 
in order to secure reliable and representative estimates of stimulus-response and response-response relationships bearing on the subception question.

\section{EXPERIMENT 2}

\section{Method}

Three veterans (J.G., T.H., D.W.) from the first experiment were retained; four subjects were added. The experienced subjects received a warm-up session with feedback following every trial: latency information concerning finger withdrawal, accuracy information about verbal report. Unpracticed subjects also received this practice with feedback, but it was followed by four additional sessions of training under actual (no feedback) experimental conditions. Then three experimental sessions per subject were conducted. At the start of each, 10 demonstration trials were shown in which a stimulus configuration was presented and identified. Next, 30 practice trials that included feedback were presented. A finger withdrawal followed by a verbal report were required on every trial, and the procedure for actual data-collection trials was identical to that used to gather the concomitant responses in Experiment 1. There were $150 \mathrm{C}+\mathrm{F}, 150 \mathrm{~F}, 30 \mathrm{~B}$, and no $\mathrm{C}$ trials in each session. All subjects were encouraged and instructed to distribute their confidence ratings over the four categories of the rating scale.

\section{Results}

It was found that two of the four novices spent eight sessions engaged essentially in a guessing task. Neither verbal reports nor reaction times gave any indication of discrimination. Data from the two who could not detect are excluded from the analysis to follow (except for inclusion in the latency data of Table 2). Suffice to say that the detection task cannot be performed by all subjects initially, and even substantial amounts of practice may prove ineffective.

For each subject, data were pooled across the three concomitant-response sessions providing $450 \mathrm{C}+\mathrm{F}$ and $450 \mathrm{~F}$ trials. The outcomes in terms of ROC curves are shown in Figure 2 for the five individuals who could detect, with composite data (the "average" detector) at the bottom. In the first column of Figure 2, the ROC curves show that, for all five subjects, the offset of the center light under metacontrast conditions was detectable by either response system. As in the first experiment, the more sensitive response system differed across subjects. For J.G., T.H., and L.F., verbal report was the more sensitive; all points lie above the reaction-time ROC curve. By Gourevitch and Galanter (1967) test, G values (treated statistically as $\mathrm{Z}$ values from the unit-normal distribution) were, respectively, $7.31,12.95$, and 5.79 , all highly significant. For D.W. and M.C., reaction time was the more sensitive system; the outcome did not meet a .05-level two-tailed test criterion for D.W. $(G=$ $-1.62)$, but did for M.C. $(G=-3.71)$. For the three subjects who served in both experiments, verbal report was consistently superior for T.H. and J.G. while reaction time remained superior for D.W.

A relatively simple detectability index is the area under a ROC curve. Each verbal-report curve was

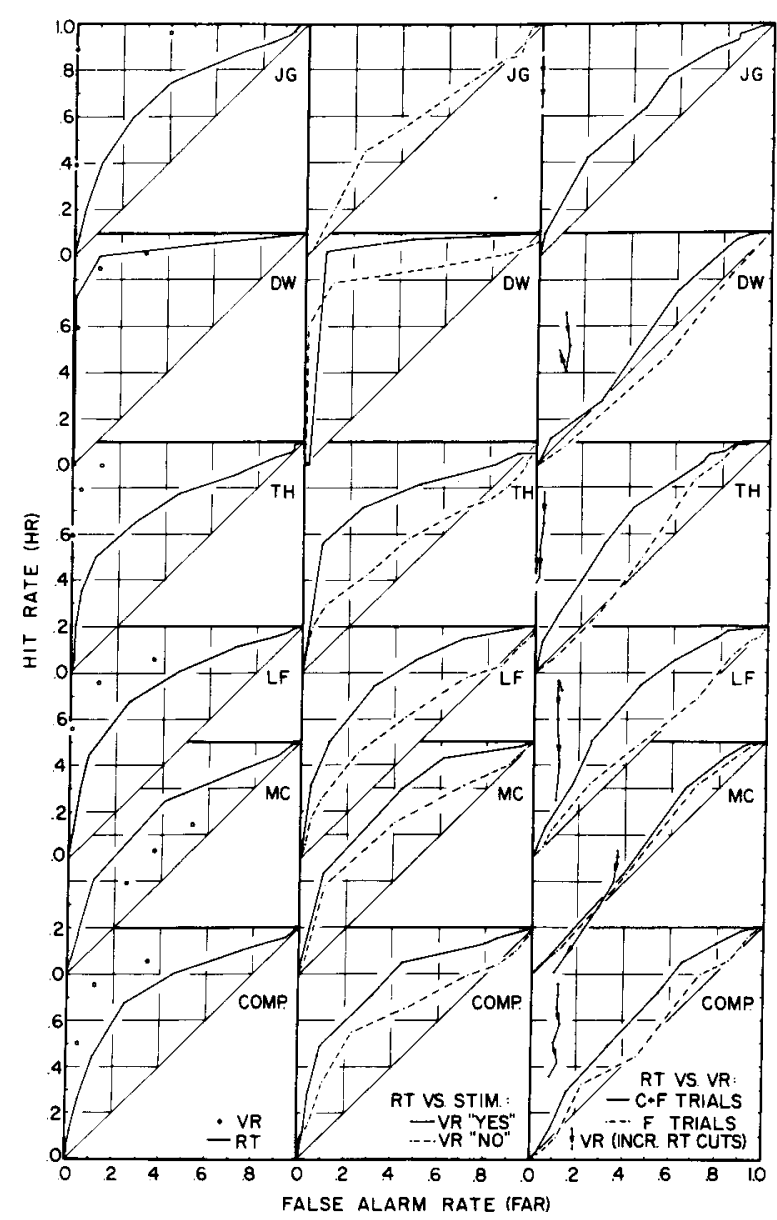

Figure 2. Selected detection data (all concomitant responses) for the five detecting subjects in Experiment 2 and the composite detecting subject.

first constructed by connecting adjacent FAR-HR data points (commencing at 0,0 and ending at 1,1 ) with straight lines. One argument against such a construction is that area is underestimated relative to an equivalent reaction-time ROC curve because latencies provide many more data points. Regardless of such difficulties, ROC-curve area is a useful summary statistic and the areas pertaining to Figure 2 are given in Table 3. Statistical tests can be performed using estimates of between-subject variability, but it must be remembered that, in addition to the five subjects listed, two whose verbal reports and response latencies gave no evidence of detectability (areas $\simeq .5$ ) are omitted.

Columns 2 and 3 of Figure 2 depict conditional ROC curves, an assessment of detectability for one response with the other held constant. Such functions are necessary for understanding the interlocking relationship among stimuli and responses. The second column of Figure 2 shows reaction-time detection of the stimulus with verbal report held constant at a positive response (treating Yes + and Yes as positive), 
Table 3

ROC-Curve Areas for Experiment 2

\begin{tabular}{|c|c|c|c|c|c|c|}
\hline \multirow[b]{2}{*}{ Subject } & \multicolumn{6}{|c|}{ Location in Figure 2} \\
\hline & \multicolumn{2}{|c|}{ Column 1} & \multicolumn{2}{|c|}{$\begin{array}{c}\text { Column } 2 \\
\text { RT vs. Stimulus }\end{array}$} & \multicolumn{2}{|c|}{$\begin{array}{l}\text { Column } 3 \\
\text { RT vs. VR }\end{array}$} \\
\hline $\begin{array}{l}\text { J.G. } \\
\text { D.W. } \\
\text { T.H. } \\
\text { L.F. } \\
\text { M.C. }\end{array}$ & $\begin{array}{l}.956 \\
.915 \\
.932 \\
.865 \\
.586\end{array}$ & $\begin{array}{l}.714 \\
.929 \\
.739 \\
.758 \\
.705\end{array}$ & $\begin{array}{l}.917 \\
.767 \\
.785 \\
.737\end{array}$ & $\begin{array}{l}.581 \\
.835 \\
.572 \\
.610 \\
.659\end{array}$ & $\begin{array}{l}.654 \\
.573 \\
.690 \\
.681 \\
.566\end{array}$ & $\begin{array}{l}.458 \\
.553 \\
.522 \\
.538\end{array}$ \\
\hline Composite & .846 & .747 & .770 & .638 & .625 & .527 \\
\hline
\end{tabular}

and also with verbal report held constant at a negative response (treating No and $\mathrm{No}+$ as negative). There are actually six functions, two associated with each verbal-report criterion location; only the two for the middle criterion cut are shown. (For J.G., under the positive verbal report, the ROC curve is indeterminate; J.G. never responded Yes + or Yes on an $F$ trial.) Statistically significant detectability was obtained for all except two ROC curves (VR negative for J.G. and for T.H.). Please note that reaction time shows greater detectability when the subject reported verbally that the stimulus was present.

In Column 3 of Figure 2, the converse relationship, verbal-report detection with reaction time held constant, is shown as connected line segments containing directional arrowheads proceeding more or less vertically. To obtain these data, a latency criterion was selected for reaction time. Then, only for trials on which the finger-withdrawal latency exceeded the criterion, the concomitant verbal reports were cast into a stimulus-response matrix. From the matrix, a verbal-report FAR-HR estimate was determined for the yes-no category boundary. Each finger-withdrawal latency criterion yields a matrix and an FAR-HR estimate. Figure 2 Column 3 shows the trend across subjects, namely, as the reaction-time criterion location moved toward longer and longer latencies (the direction of the arrows), hit rates declined while false alarm rates tended to remain stable. These outcomes are statistically significant. Each latency criterion actually determines two verbal-report matrices, one associated with longer and one with shorter latencies; each matrix has three verbal-report criterion locations that can be computed. Only the most informative of the six possible functions was chosen for plotting.

Also in Column 3 of Figure 2 is shown the relationship between response systems holding the stimulus configuration constant (so-called Type II ROC curves, Green \& Swets, 1966, p. 302). As one example, the stimulus can be held constant by selecting $\mathrm{C}+\mathrm{F}$ trials only, comparing reaction times when verbal report falls in either category of yes to reaction times when verbal report falls in either category of no, the solid-line ROC curve in the figure. The "hit rate" stands for the probability of a rapid finger response given that the subject responded with a yes, the "false-alarm rate" is the probability of a rapid response given a no. For $\mathrm{C}+\mathrm{F}$, but not for $\mathrm{F}$ trials, verbal report and reaction time are related such that, when the subject claimed to detect the blink (said yes), reaction times were likely to be quicker. (The $C+F$ functions are statistically significant from zero detectability for all subjects, the F function only for M.C. The functions associated with the other verbal-report criterion cuts are not shown. For J.G., the ROC curve for $\mathrm{F}$ trials is again indeterminate.)

The calculation of an average subject-composite subject is perhaps a better term-was considered to be worthwhile despite the problems with aggregating detection data. The practice of casting the data for all subjects into one confusion matrix practically guarantees that detectability "averaged across subjects" will be underestimated. The data for the composite subject shown at the bottom of Figure 2 were combined differently. A suitable linear transformation $(\mathrm{ax}+\mathrm{b})$ was applied to all the latencies of $\mathrm{a}$ given subject to set his transformed means for $\mathrm{C}+\mathrm{F}$ trials and $\mathrm{F}$ trials into coincidence with the respective $\mathrm{C}+\mathrm{F}$ and $\mathrm{F}$ means for the group. The transformations do not alter the ROC curves of any subject, but they act to reduce between-subject variance that makes aggregate performance appear worse than it ought. The transformed data were cast into a 2 $(C+F, F)$ by 4 (Yes +, Yes, No, No +) by 46 (latencies in 10-msec class intervals) matrix. Mean response frequencies were obtained by dividing the cell entries by 5 (collapsing adjacent latency categories where necessary) to obtain a composite subject who had served, like a real subject, for 900 trials. For the composite subject, verbal report won handily over reaction time (Gourevitch \& Galanter $G=5.17$ ) as the ROC data at the bottom of Column 1, Figure 2 attest. All contingent detectabilities were also significantly above chance detectability (.05 level) except that RT vs. VR for F trials was not (bottom of Column 3). Reaction times before transforming are listed in Table 2; the additional practice has speeded up performance in the second experiment compared to the first.

\section{DISCUSSION}

The Fehrer and Biederman (1962) modification of the traditional metacontrast experiment met all expectations as a suitable subception paradigm. The first experiment established that the choice of stimulus values, including the 35 -msec delay between center-light offset and flanking-lights onset, was appropriate. Simple reaction time proved to be a con- 
sistent detection system for many subjects, even in the face of cognitive uncertainty about the nature of the stimulus events as shown by confidence ratings and subjects' informal observations.

Taken together, the experiments highlight large individual differences that do not disappear with practice. In addition to the 8 subjects discussed, 6 other subjects completed the first three training sessions of Experiment 1. Of these 14 who completed a minimum of three sessions, 3 subjects failed to demonstrate any detection, 2 favored reaction time as the superior response mode, 6 favored verbal report, and 3 were indeterminate. The authors, at least, are satisfied with their decision to hold stimulus parameters fixed rather than tailor them to each subject for the following reasons: A difficult decision that did not need to be faced was which response to try to control. Nulling one aspect of performance across subjects would still leave others uncontrolled. Information about individual differences would have been lost, and the range of performance was large, with some subjects showing chance detection after many hours of practice. (Learning does occur. The figures document improvements in detection by the three subjects who served in both experiments. Incidentally, J.G. gave no evidence of detection until the fourth practice session.) Finally, the patterns of stimulus-response and response-response relationships revealed in Figure 2 are consistent across subjects, enough so for us to argue that the composite subject is more than a convenient fiction.

The present data may be compared with those of the Bernstein et al. (1973) experiment in which a single-response signal-detection analysis was applied to the Fehrer and Biederman (1962) metacontrast design. Table 2 shows that with respect to metacontrast latencies, reaction-time latency to the offset of the center light alone $(C)$ is slower than the latency to the masked offset $(C+F)$, and slower even than to the onset of the masks alone (F), which occurred $35 \mathrm{msec}$ later. The latency data for nonconcomitant finger responses (Experiment 1) are in very close agreement but slightly longer than the equivalent data from Bernstein et al. (interpolating to estimate a stimulus onset asynchrony of $35 \mathrm{msec}$ from their data). The latency increase might depend on the fact that in the present experiments, unlike Bernstein et al., exceptionally long or short latencies were not replaced with new data. (The effect of deviant latencies was to increase the mean reaction times in Table 2.) Setting aside deviant latencies in our data would have given an unfair detection advantage to finger responses. Furthermore, in evaluating any response system, every response, good or bad, is relevant in determining the performance of the system.

Note from Table 2 that reaction times in Experiment 1 were demonstrably faster when no verbal re- port was required. However, detectability did not also improve (cf. RT curves in Figure 1). Quick subjects were not better detectors than slow subjects. For reaction-time data, response latency and discriminability (measured by area under the ROC curve) were not correlated significantly across 10 subjects on their 3rd day of practice in Experiment 1. By far the best reaction-time detection was shown by D.W., who was third in average response latency among the five detectors in Experiment 2.

Which response system is the more sensitive? Bernstein et al. (1973), for nonconcomitant responding, obtained an average detection superiority (four subjects) of verbal report over reaction time at every level of stimulus onset asynchrony. Taken in the aggregate, our concomitant and nonconcomitant data for the one stimulus asynchrony tested concur, but it must be reemphasized that the average hides severe individual differences. Some subjects (3 of 14) cannot perform at all, and for others (2, perhaps 3 ), reaction time, the nonverbal response, is the better.

What is the relationship between the two response systems? There are ROC-curve equivalents to the correlation approach. As the area under the ROC curve exceeds .5 , the degree of positive relationship between variables, like response and stimulus, increases. When the area reaches 1.0, the area of the unit square, responses are capable of predicting stimuli without error, given proper criterion placement. Letting RT stand for reaction time, VR for verbal report, and ST for stimulus, the three correlations, the three partial correlations, and their ROC-curve equivalents shown in Figure 2 are:

$\mathrm{r}_{\mathrm{RT}, \mathrm{ST}}$-The reaction-time ROC curves (Column 1).

rVR,ST-The three points for verbal-report criterion cuts (Column 1).

$r_{R T, V R}-$ Not shown, and of less interest because the type of stimulus is ignored. Faster reaction times were associated slightly with yes responses.

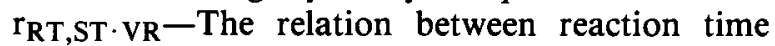
and the stimulus holding verbal report constant (Column 2). Reaction time conveyed information about the stimulus for a verbal response of yes or no. (Within each of the four verbal-report categories, reaction times carried information about the stimulus, in support of the existence of a partial correlation.)

$\mathrm{r}_{\mathrm{VR}, \mathrm{ST} \cdot \mathrm{RT}}$ - The relation between verbal report and the stimulus holding reaction time constant (Column 3). Shown are FAR-HR pairs corresponding to selected reaction-time criterion cuts. Verbal report declined in detectability as reaction times increased.

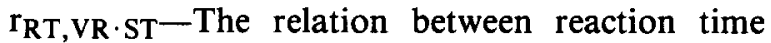
and verbal report with the stimulus configuration held constant (Column 3 ). Reaction times and verbal report were related only for $\mathrm{C}+\mathrm{F}$ stimulus trials.

Since the data of both experiments showed that, on the average, verbal reports conveyed more informa- 
tion about the stimulus than did reaction times ( $r_{V R, S T}$ exceeded $r_{R T, S T}$ ), then, in overall detectability, the nonverbal response system was inferior. However, even when verbal report is partialed out, reaction time still provides information about the

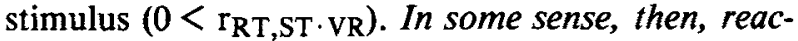
tion time can be said to be carrying information not available to verbal report. Conversely, verbal report conveys information when reaction time is partialed out $\left(0<r_{V R, S T} \cdot R T\right)$. Therefore, predictions about the stimulus would be improved by using a properly weighted combination of the two responses rather than by using the more sensitive one alone.

\section{A MODEL OF EXPERIMENT 2}

The ROC curves in Figure 2 for the composite subject can be conceived of as based upon various criterion cuts through the matrix of the aggregate data ( 2 stimulus configurations by 4 verbal-report categories by a large number of reaction-time categories). The matrix itself can be represented graphically in the same manner that correlation scatterplots are depicted as bivariate surfaces in outline on a plane. Such a representation is shown in Figure 3. Reaction time is plotted on the abscissa; the empirical data for the composite subject were used. Verbal-report distributions, which must be inferred, lie on the ordinate. The usual assumptions of overlapping normal distributions with equal variance were employed in characterizing verbal report. (The fit of the three empirical verbal-report criterion points with respect to these assumptions was checked, and it is adequate.) Distances along abscissa and ordinate have been equated with respect to the standard deviations of their respective responses. The bivariate distributions, egg shaped in outline, depict the relationship of verbal report to reaction time given the F stimulus configuration and given the $\mathrm{C}+\mathrm{F}$ configuration. Category boundaries for verbal reports appear as horizontal line segments, which require the additional assumption that the boundaries are independent of reaction time. The verbal-report means across reaction-time latencies appear as dot-dash lines. Verbal report and reaction times are correlated under $\mathrm{C}+\mathrm{F}$ conditions only. For very-short-latency reaction times, verbal-report detectability is reduced (less difference between the $\mathrm{C}+\mathrm{F}$ and $\mathrm{F}$ means), is maximum near $200 \mathrm{msec}$ (greatest difference between means), and as reaction-time latencies grow longer and longer, verbal-report detectability declines apace (the means converge). Figure 3 is another way to represent the information presented by the ROC curves for the composite subject of Figure 2. For metacontrast, Figure 3 is the pictorial solution to the correlation approach to subception discussed by Eriksen

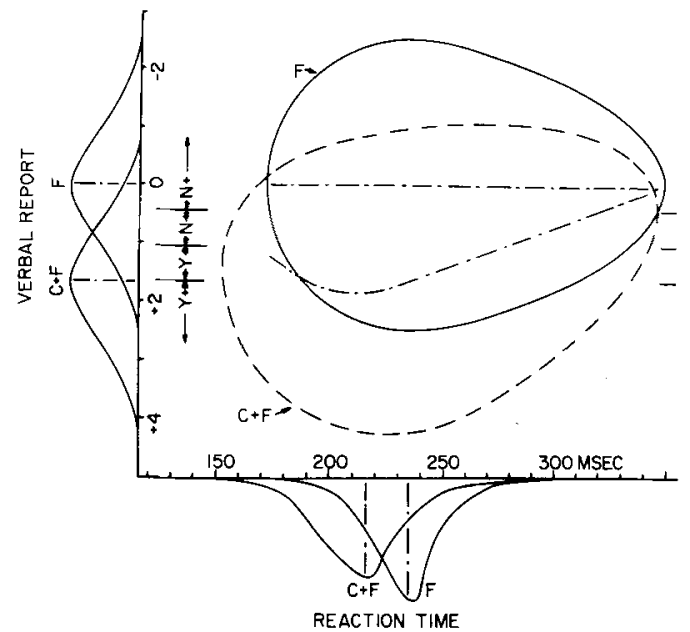

Figure 3. A bivariate model of the data of Experiment 2 based upon the composite subject.

(1956, 1957, 1960), given that the assumptions associated with the hypothetical verbal-report distributions are tenable.

Chun and Sarbin (1968) emphasized that skinresistance measures taken concurrently with verbal reports may reflect both the covert decision-making process and the overt report. Similarly, with finger withdrawal, one possibility is that the decision process leading ultimately to a verbal report is made quickly and reaction-time latency mirrors the decision. This would imply that reaction times cannot predict the stimulus with verbal report held constant, i.e., $\mathrm{r}_{\mathrm{RT}, \mathrm{ST} \cdot \mathrm{VR}}=0$. However, reaction times always provide information about the stimulus for any verbalreport category. Conversely, verbal reports might depend directly upon the latency of reaction time, making rVR ST.RT $_{\text {V }}=0$. Such was not the case. Therefore, the serial-response model of subception, in which one response system is fed not by information from the visual system, but directly by the output of the other response system, can be rejected.

The data do support a parallel-response model (Eriksen, 1960). The stimulus leads to a perceptual process, and each response system independently taps the output of perceptual processing.

$$
\text { Stimulus } \rightarrow \text { perceptual process } \overbrace{\text { verbal report }}^{\text {simple reaction time }}
$$

Errors introduced by one response system will be uncorrelated with errors introduced by the other. Response systems were not correlated for $\mathrm{F}$ trials; why were they correlated for $\mathrm{C}+\mathrm{F}$ trials? The following is a relatively straightforward explanation. Suppose that, prior to the completion of perceptual processing 
and before the response systems are activated, information about the signal, the of fset of the center light in a $\mathrm{C}+\mathrm{F}$ trial, is distorted or lost. The onset of the flanking lights is a powerful stimulus event, which the subject can hardly fail to process. The offset of the center light is a mild psychological event because of its brevity and because of metacontrast. When the center light does go off, the subject may or may not process the offset. Therefore, the latency distribution for $\mathrm{C}+\mathrm{F}$ trials can be considered as a mixture of trials on which the offset was responded to, and on which the offset was missed but the flanks were responded to. (Observe, from Figure 3, that the standard deviation of $\mathrm{C}+\mathrm{F}$ latencies is larger than that of $F$ latencies even though $F$ trials have a longer average latency.) The simple principles are that (a) missed signals are missed perceptually, (b) missed signals must occur on $\mathrm{C}+\mathrm{F}$ trials, and (c) the perceiver never misses a flanking light. Signals missed perceptually lead both to a higher proportion of "no-signal" verbal responses and to longer latency reaction times, explaining the correlation between response systems on $\mathrm{C}+\mathrm{F}$ trials. Stated from the response end, the longer the latency on a $\mathrm{C}+\mathrm{F}$ trial, the more likely it is that the perceiver has missed the signal. A model postulating that subjects from time to time do the processing equivalent of closing their eyes is totally inappropriate because flanking lights were never ignored. For the seven subjects in Experiment 2, which includes the two nondiscriminating subjects, the longest response for all 6,300 finger withdrawals was $550 \mathrm{msec}$.

The reduced verbal-report detectability at very short reaction-time latencies requires separate treatment. Very rapid finger withdrawals may be interpreted as anticipations containing no information about the state of the world. Verbal reports, which always occur long after the stimulus presentation, should not necessarily suffer a decrement, but they do. Perhaps the initiation of an anticipatory finger withdrawal tends to disrupt perceptual processing so that verbal reports are also affected.

The analysis permitted us to ascribe some of the perturbations in the responses to perceptual processing, because both response systems must reflect the distortions added by processing of the input. The errors uncorrelated between response systems can be attributed to variability added by each system. For metacontrast, let us modify the parallel-response model proposed by Eriksen (1960) by inserting a decision stage concerning the stimulus into the chain leading to verbal report:

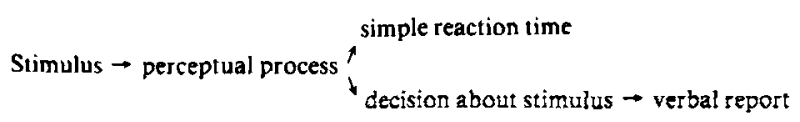

In signal-detection terms, decision processes are involved in maintaining a criterion (or criteria) and comparing the outcome of perceptual processing with that criterion. The inability to maintain stable decision criteria adds variance that reduces detectability. In fact, the "response" variability inherent in verbal-report measures may be primarily variability introduced by decision making. Any verbalization ought to bear a near-perfect relationship to the decision that precedes it. For reaction time, no decisions are made, which eliminates a potential source of variance, but rapid motor movements have their own sources of variance.

A decent account of the data has been provided, but what of subception? Subception, it seems to us, requires that the response system acting below the level of awareness have access to information about the world that is unavailable to the response system linked to consciousness. The most plausible conclusion based upon the metacontrast evidence is that neither response system has a private supply of information. Any gain in predictability of the stimulus from dual responding results from taking advantage of uncorrelated response variability. Unfortunately, even when reaction-time detection is superior to verbal report, as it is for a few subjects, the claim can always be made that, rather than the finger response having access to additional information, its variability is less than that of verbal report.

\section{SUMMARY}

A difficult detection task was chosen in which perceivers have moderate to severe cognitive uncertainty about the state of the world, and it was shown that additional predictability can be gleaned from a concomitant nonverbal response. Some will label this subception. Subception proponents may overlook the reciprocal fact that the concomitant verbal response also supplies additional predictability. The theorist must grant the possibility that nonoverlapping "information" to be found in both the conscious and the unconscious response is the result of uncorrelated response variability rather than of privileged access to the outside world. Demonstrating better detection for the nonverbal response is not convincing support for subception, because reduced response variability can be offered as an alternative interpretation. The clincher would have been to find evidence that verbal report was concomitantly operating at chance levels when the nonverbal response gave evidence of detection. The signal-detection approach to subception, by avoiding troublesome aspects of response bias and thresholds, provides the rules for a fair fight. Our choice of stimulus parameters in the modified metacontrast paradigm based 
upon previously published performance levels gave hopes that, if convincing support for subception were to be found, we would have found it. Count us among the former optimists.

\section{REFERENCES}

Bernstein, I. H., Amundson, V., \& Schurman, D. L. Metacontrast inferred from reaction time and verbal report. Replication and comments on the Fehrer-Biederman experiment. Journal of Experimental Psychology, 1973, 100, 195-201.

Chun, K.-T., \& Sarbin, T. R. Methodological artifacts in subception research. Psychological Record, 1968, 18, 137-149.

Dulany, D. E., JR., \& Eriksen, C. W. Accuracy of brightness discrimination as measured by concurrent verbal responses and GSRs. Journal of Abnormal and Social Psychology, 1959, 59, 418-423.

Eriksen, C. W. Subception: Fact or artifact? Psychological Review, 1956, 63, 74-80.

Eriksen, C. W. Prediction from and interaction among multiple concurrent discriminative responses. Journal of Experimental Psychology, 1957, 53, 353-359.

ERIKSEN, C. W. Discrimination and learning without awareness: A methodological survey and evaluation. Psychological Review, $1960,67,279-300$.
Fenrer, E., \& Biederman, I. A comparison of reaction time and verbal report in the detection of masked stimuli. Journal of Experimental Psychology, 1962, 64, 126-130.

Fehrer, E., \& RAAB, D. Reaction time to stimuli masked by metacontrast. Journal of Experimental Psychology, 1962, 63, 143-147.

Gourevitch, V., \& Galanter, E. A significance test for one parameter isosensitivity functions. Psychometrika, 1967, 32, 25-33.

Green, D. M., \& Swets, J. A. Signal detection theory and psychophysics. New York: Wiley, 1966.

Lazarus, R. S., \& McCleary, R. A. Autonomic discrimination without awareness: A study of subception. Psychological Review, 1951, 58, 113-122.

RAAB, D. H. Backward masking. Psychological Bulletin, 1963, 60, 118-126.

Schiller, P. H., \& Smith, M. C. Detection in metacontrast. Journal of Experimental Psychology, 1966, 71, 32-39.

Swets, J. A., Tanner, W. P., Jr., \& Birdsall, T. G. Decision processes in perception. Psychological Review, 1961, 68, 301-340.

(Received for publication March 6, 1979; revision accepted June 13, 1979.) 